\title{
Recent advances in mast cell clonality and anaphylaxis
}

\author{
Cem Akin
}

\author{
Address: Brigham \& Women's Hospital, Division of Rheumatology, Immunology \& Allergy, 1 Jimmy Fund Way, Smith Building, \\ Room 626B, Boston, MA 02445, USA \\ Email: cakin@rics.bwh.harvard.edu
}

FI000 Medicine Reports 2009, I:97 (doi:10.3410/MI-97)

The electronic version of this article is the complete one and can be found at: http://FI000.com/Reports/Medicine/content///97

\begin{abstract}
Clonal expansion of mast cells carrying the D8I6V c-kit mutation results in mastocytosis. Recent studies identified the presence of clonal mast cells carrying this mutation in patients with anaphylaxis without classic diagnostic findings of systemic mastocytosis.
\end{abstract}

\section{Introduction and context}

Since the description of the first patient with systemic mastocytosis, the gold standard of diagnosing this disorder has been the demonstration of increased mast cells in clusters in tissue biopsies, most commonly of the bone marrow [1]. Therefore, the term mast cell disease has been used synonymously with mast cell proliferative disorders. Patients with systemic mastocytosis not only have increased mast cells in tissue biopsies but also have elevated serum levels of tryptase (greater than $20 \mathrm{ng} / \mathrm{mL}$ ), a mast cell protease used as a surrogate marker of mast cell burden (Table 1). Molecular mechanisms resulting in the accumulation of mast cells in mastocytosis have not been completely elucidated; however, a gain-of-function mutation in c-kit (D816V) is found in almost all patients with systemic mastocytosis [2]. This mutation renders the Kit tyrosine kinase independent of its ligand stem cell factor, resulting in constitutive autophosphorylation and activation of downstream signal transduction cascades. Kit is expressed in large quantities on mast cell membrane and functions to induce differentiation and proliferation of mast cell progenitors, protect them from apoptosis, and enhance mast cell activation [3].

\section{Recent advances}

Patients with mastocytosis are at higher risk for developing hypotensive episodes resembling anaphylaxis [4]. A recent study estimated the incidence of anaphylaxis to be as high as $49 \%$ in patients with mastocytosis, representing approximately a 1000-fold increase in incidence compared with the general population [5]. The most common etiologic factors of anaphylaxis are drugs, foods, exercise, and Hymenoptera stings. However, an etiology cannot be identified in a significant proportion of patients referred to allergists after one or more episodes of anaphylaxis [6]. These patients are termed to have idiopathic anaphylaxis. Based on the observation that mast cell disease is associated with increased risk of anaphylaxis, patients with unexplained anaphylaxis have been studied for the presence of aberrant clonal bone marrow mast cells as seen in mastocytosis. One study found that 5 out of 12 patients with recurrent anaphylaxis had aberrant mast cells as identified by such markers as the c-kit D $816 \mathrm{~V}$ mutation or the expression of CD25 surface marker [7]. Interestingly, these patients did not have any apparent diagnostic evidence of a proliferative mast cell disease such as clusters of mast cells in bone marrow or urticaria pigmentosa skin lesions. The demonstration of aberrant mast cells in these patients required sensitive techniques of flow cytometry or mutation analysis of enriched mast cells. Thus, these patients presented with a disorder characterized primarily by activation rather than proliferation of mast cells, despite carrying the same c-kit mutation in systemic mastocytosis. Such patients who lack major diagnostic criteria of mastocytosis (multifocal aggregates of mast cells in tissue biopsies) but who are detected to have 
Table I. Diagnostic criteria for systemic mastocytosis

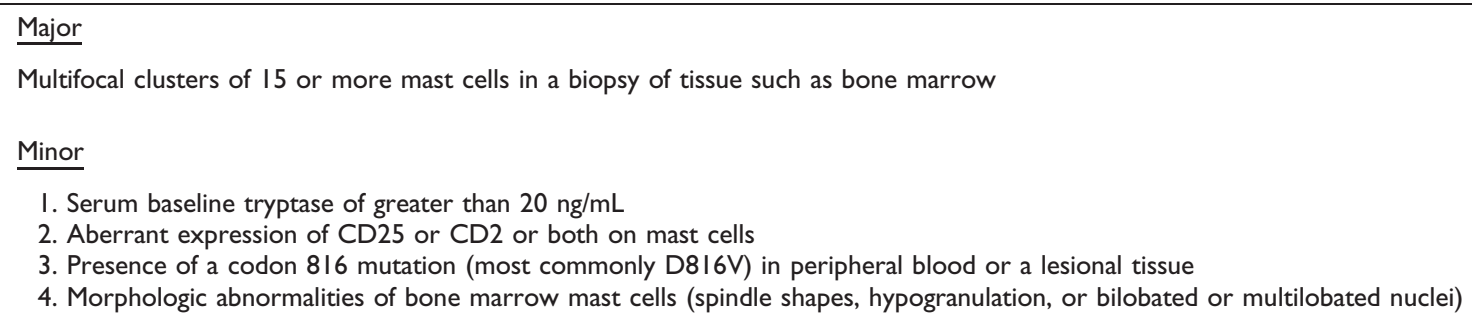

Either one major and one minor or three minor criteria are needed to make a diagnosis of systemic mastocytosis.

one or two minor diagnostic criteria (c-kit mutation, CD25 expression, or morphologic abnormalities such as spindle shapes and hypogranulation) are termed to have 'monoclonal mast cell activation syndrome' (MMAS) $[8,9]$. Some patients with MMAS have normal or only slightly elevated serum tryptase levels, making it especially challenging to diagnose a mast cell disorder. From the hematopoietic clonal standpoint, this nomenclature is perhaps analogous to monoclonal gammapathy of undetermined significance, which represents a plasma cell disorder with a limited clonal expansion. Follow-up of these patients so far has suggested that most do not progress to systemic mastocytosis (personal observation).

Recent studies examined the role of mast cell clonality in patients who experience anaphylaxis after Hymenoptera stings [10]. In a study examining 379 patients with systemic reactions to Hymenoptera stings, it was found that approximately $12 \%$ have elevated baseline tryptase levels [11]. Approximately $62 \%$ of these patients with elevated tryptase levels satisfied the criteria for systemic mastocytosis, whereas $26.5 \%$ had MMAS. The incidence of clonal mast cell disease in patients who experience anaphylaxis to other triggers such as drugs and foods has also been investigated and found to be approximately $1.5 \%$, while $6.6 \%$ of this population had elevated tryptase levels [12]. Clinical analysis of symptomatic presentation of patients with clonal mast cell disease showed that they suffered significantly more hypotensive episodes in the absence of urticaria as compared with patients with true idiopathic anaphylaxis [13].

\section{Implications for clinical practice}

Based on these recent findings, we recommend the investigation of occult or overt mast cell disease in all patients with recurrent hypotensive anaphylaxis episodes, especially those with idiopathic anaphylaxis and Hymenoptera sensitivity. A baseline tryptase level should be obtained at a minimum in addition to the usual workup to determine an allergic etiology. Patients with elevated baseline tryptase levels should be referred to a specialized center to have clonal mast cell disease investigated.

Clinical management of patients with clonal mast cell disease includes symptomatic therapy with antimediator medications such as antihistamines, antileukotriene medications, glucocorticoids, and cromolyn individualized for each patient. Self-injectable epinephrine should be considered for every patient due to the increased risk of anaphylaxis. Patients with a history of Hymenoptera anaphylaxis should be referred to an allergist for evaluation of IgE-mediated sensitization. If found to have allergic Hymenoptera sensitization, patients with clonal mast cell disease are candidates for lifelong Hymenoptera venom immunotherapy.

\section{Abbreviation}

MMAS, monoclonal mast cell activation syndrome.

\section{Competing interests}

The author declares that he has no competing interests.

\section{References}

I. Metcalfe DD: Mast cells and mastocytosis. Blood 2008, I | 2:946-56.

2. Akin C: Molecular diagnosis of mast cell disorders: a paper from the 2005 William Beaumont Hospital Symposium on Molecular Pathology. J Mol Diagn 2006, 8:4I2-9.

3. Jensen BM, Akin C, Gilfillan AM: Pharmacological targeting of the KIT growth factor receptor: a therapeutic consideration for mast cell disorders. Br J Pharmacol 2008, I 54: I572-82.

4. Muller UR, Haeberli G: The problem of anaphylaxis and mastocytosis. Curr Allergy Asthma Rep 2009, 9:64-70.

5. Brockow K, Jofer C, Behrendt H, Ring J: Anaphylaxis in patients with mastocytosis: a study on history, clinical features and risk factors in 120 patients. Allergy 2008, 63:226-32.

6. Webb LM, Lieberman P: Anaphylaxis: a review of 601 cases. Ann Allergy Asthma Immunol 2006, 97:39-43.

7. Akin C, Scott LM, Kocabas CN, Kushnir-Sukhov N, Brittain E, Noel P, Metcalfe DD: Demonstration of an aberrant mast-cell population with clonal markers in a subset of patients with 'idiopathic' anaphylaxis. Blood 2007, I I 0:233 I-3.

8. Sonneck K, Florian S, Müllauer L, Wimazal F, Födinger M, Sperr WR, Valent P: Diagnostic and subdiagnostic accumulation of mast cells in the bone marrow of patients with anaphylaxis: monoclonal mast cell activation syndrome. Int Arch Allergy Immunol 2007, 142:158-64. 
9. Valent P, Akin C, Escribano L, Födinger M, Hartmann K, Brockow K, Castells M, Sperr WR, Kluin-Nelemans HC, Hamdy NA, Lortholary O, Robyn J, van Doormaal J, Sotlar K, Hauswirth AW, Arock M, Hermine O, Hellmann A, Triggiani M, Niedoszytko M, Schwartz LB, Orfao A, Horny HP, Metcalfe DD: Standards and standardization in mastocytosis: consensus statements on diagnostics, treatment recommendations, and response criteria. Eur J Clin Invest 2007, 37:435-53.

10. Rueff F, Placzek M, Przybilla B: Mastocytosis and Hymenoptera venom allergy. Curr Opin Allergy Clin Immunol 2006, 6:284-8.

II. Bonadonna P, Perbellini O, Passalacqua G, Caruso B, Colarossi S, Dal Fior D, Castellani L, Bonetto C, Frattini F, Dama A, Martinelli G, Chilosi M, Senna G, Pizzolo G, Zanotti R: Clonal mast cell disorders in patients with systemic reactions to
Hymenoptera stings and increased serum tryptase levels. J Allergy Clin Immunol 2009, 123:680-6.

Changes Clinical Practice

FI000 Factor 6.0 Must Read

Evaluated by Cem Akin 04 Jun 2009

12. Bonadonna P, Zanotti R, Pagani M, Caruso B, Perbellini O, Colarossi S, Olivieri E, Dama A, Schiappoli M, Senna G, Antico A, Passalacqua G: How much specific is the association between Hymenoptera venom allergy and mastocytosis? Allergy 2009, 64:1379-82.

13. Koterba A, Akin C: Differences in the clinical presentation of anaphylaxis in patients with indolent systemic mastocytosis (ISM) versus idiopathic anaphylaxis (IA). J Allergy Clin Immunol 2008, I 2 I (Suppl I):S68-9. 